\title{
EJERCICIO Y QUEMA DE GRASA: \\ ¿COMER O NO COMER ANTES DE ENTRENAR?
}

\author{
EXERCISE AND FAT BURNING: \\ ¿TO EAT OR NOT TO EAT BEFORE TRAINING?
}

\author{
Norman MacMillan K. \\ Escuela de Educación Física, Pontificia Universidad Católica de Valparaíso. Chile.
}

\begin{abstract}
The World Health Organization (WHO) has proposed to associate diet and exercise to handle the overweight prevalent in the population. The traditional exercise recommendation for fat burning is a moderate intensity session. In this condition fat oxidation during the activity reaches 6 to 8 grams per minute, which is greatly diminished when previous carbohydrates is ingested. Another option recently studied as a strategy for managing overweight is strength training or intermittent high intensity efforts. In this condition glycogen depletion favors the use of adipose tissue as energy after the exercise. With this strategy it is not necessary fasting before training. Carbohydrates ingestion prior to the session will increase glycogen oxidation and promote greater fat oxidation after the exercise.
\end{abstract}

Key words: exercise, fat burning, fast state.

Este trabajo fue recibido el 10 de Diciembre de 2007 y aceptado para ser publicado el 15 de Diciembre de 2008.

\section{INTRODUCCIÓN}

La Organización Mundial de la Salud (OMS) y la mayoría de las organizaciones médicas, recomiendan asociar la dieta y la actividad física para promover la salud y reducir la creciente incidencia de obesidad en la población mundial (1-3). Conscientes de esta evidencia, sujetos con sobrepeso se incorporan a programas de ejercicio orientado a reducir su adiposidad. Luego de mejorar la condición física y la capacidad de entrenamiento, muchos buscan además modificaciones en la dieta que optimicen los cambios en su estructura corporal. Una de las estrategias que se ha planteado de utilidad para incrementar la "quema" de grasa es realizar un ejercicio de intensidad moderada y el ayuno previo a la sesión $(4,5)$. En este artículo se revisa la evidencia científica respecto a las estrategias de ejercicio y la influencia de la ración alimentaria previa a la sesión, en la utilización de sustratos energéticos y su importancia en un programa de control de peso para sujetos físicamente activos.

\section{EJERCICIO AERÓBICO Y AYUNO}

La energía utilizada durante el ejercicio proviene de una mezcla de grasa y carbohidratos. En una sesión de 30 a 60 minutos de duración, con una intensidad ideal para la utilización de grasa, que corresponde aproximadamente a un $75 \%$ de la frecuencia cardiaca máxima, cerca de la mitad de la energía utilizada proviene de las reservas de triglicéridos corporales, lo que implica una oxidación de 0.5 a 0.8 gramos de grasa por minuto $(6,7)$.

Se ha demostrado que la ingesta de carbohidratos en una ración previa a un esfuerzo de esta intensidad, puede inhibir tanto la liberación de ácidos grasos desde el tejido adiposo (lipólisis) como su posterior uso como energía (oxidación) a nivel muscular (5-8).

En un estudio realizado con deportistas entrenados que pedalearon 40 minutos a baja intensidad $(50 \%$ de su consumo máximo de oxígeno), la ingesta de 200 gramos de carbohidratos en la hora previa al ejercicio redujo en $34 \%$ la oxidación total de grasa durante el esfuerzo (8). Otro estudio realizado con sujetos activos que pedalearon una hora a intensidad moderada, comparó la lipólisis y oxidación de grasa durante el ejercicio en estado de ayuno o luego de ingerir una solución de aproximadamente 60 gramos de glucosa o fructosa, una hora previo al ejercicio. La ingesta de glucosa redujo la oxidación de grasa en un 50\% y la de fructosa en $30 \%$ (5). Con lapsos mayores ( 3 horas) entre la alimentación y el ejercicio, se ha descrito que la ingesta de carbohidratos de alto índice 
glicémico (cereales, pan blanco) genera una reducción en la movilización de los triglicéridos del tejido adiposo a pesar que la glicemia y las concentraciones de insulina retornen a niveles basales. Estos cambios metabólicos pueden persistir incluso por hasta 6 horas luego de consumido el alimento (4).

\section{EJERCICIO DE ALTA INTENSIDAD Y AYUNO}

Recientemente se ha prestado interés al efecto de sesiones de ejercicio de alta intensidad en la reducción de tejido adiposo $(9,10)$. Durante una sesión de fortalecimiento muscular (con pesos libres o máquinas) o un ejercicio dinámico con intervalos de alta intensidad (como los realizados en deportes de equipo), la mayor parte de la energía utilizada durante el esfuerzo proviene de las reservas de glucógeno, sin embargo con reservas reducidas de carbohidratos e incremento de los niveles de catecolaminas, el organismo favorecerá la utilización de grasa como energía en el periodo posterior al ejercicio (11).

Favorecido por mecanismos similares, se ha demostrado que una rutina de trabajo con pesas (en la que se reduzcan las reservas de glucógeno e incrementen los niveles de catecolaminas) seguida de ejercicio aeróbico, es capaz de generar un incremento cercano al $100 \%$ en la oxidación de grasa durante la sesión aeróbica, en relación al ejercicio aeróbico aislado (12).

En todas estas situaciones, el incremento en la oxidación de grasa no se produce durante el ejercicio de alta intensidad sino posterior a el, por lo que no es necesario el ayuno previo. Por el contrario, el consumo de una ración moderada (20 a 30 gramos) en carbohidratos anterior a la sesión es un factor favorable al condicionar una mayor glicemia, mayor potencia de trabajo muscular, mayor utilización de glucógeno durante el ejercicio, promoviendo luego una mayor oxidación de grasa $(4,11,13)$. Ingerir una merienda previo a un entrenamiento vespertino, puede ser entonces absolutamente compatible con un programa para reducir tejido adiposo, cuando la sesión involucre predominantemente o se inicie con ejercicio de alta intensidad.

\section{CONCLUSIONES Y RECOMENDACIONES}

La ingesta de carbohidratos o el ayuno previo a la sesión, esta condicionada al tipo de ejercicio elegido en un programa para "quema" de grasa.

Cuando se utiliza el tradicional ejercicio de intensidad moderada, la ingesta previa de carbohidratos, incluso en bajas cantidades y especialmente si es de alto índice glicémico, reduce en forma importante la oxidación de grasa durante el ejercicio, por lo que la recomendación es el ayuno. Esta estrategia debe ser discutida con el de- portista y aplicada cuando la primera prioridad es reducir tejido adiposo, ya que a pesar de las potenciales ventajas en la quema de grasa, el ayuno plantea inconvenientes como favorecer el catabolismo proteico, reducción del rendimiento deportivo y el riesgo de hipoglicemia durante el esfuerzo $(1,4,5,7,14,15)$.

Cuando en la sesión predomina o se inicia con ejercicios de alta intensidad, no es necesario el ayuno previo a la sesión ya que no se oxida grasa durante el ejercicio sino después. Una ración anterior moderada en carbohidratos es más bien recomendable para incrementar la oxidación de glucógeno durante el ejercicio y consecuentemente mayor oxidación de tejido adiposo posterior al esfuerzo. $(12,13)$.

Buscando una mayor efectividad en un programa reductivo, los sujetos con sobrepeso deberían entonces elegir su estrategia de alimentación previa al ejercicio, según la intensidad que será utilizada durante la sesión de entrenamiento.

\section{RESUMEN}

La Organización Mundial de la Salud (OMS) ha propuesto asociar la dieta con el ejercicio para manejar el sobrepeso de la población. La recomendación tradicional de ejercicio para "quema" de grasa es una sesión de intensidad moderada. En esta condición la oxidación de grasa durante el esfuerzo puede llegar a 6-8 gramos por minuto, la que se reduce en forma significativa con una ingesta previa de carbohidratos. Otra opción recientemente estudiada como estrategia para el manejo del sobrepeso es el ejercicio de fortalecimiento muscular o esfuerzos intermitentes de alta intensidad. En esta condición la depleción glucogénica favorece la utilización del tejido adiposo como energía después de la sesión. Con esta estrategia no es necesario el ayuno previo e incluso es recomendable una ración de carbohidratos antes de la sesión para incrementar la oxidación de glucógeno durante el ejercicio, una mayor depleción y favorecer una mayor oxidación de tejido adiposo luego del esfuerzo.

Palabras claves: ejercicio, quema de grasa, ayuno.

Dirigir la correspondencia a:

Dr.

Norman MacMillan

Escuela de Educación Física,

Pontificia Universidad Católica de Valparaíso

Av. El Bosque 1290,

Santa Inés, Viña del Mar.

Fono: 32-2274377

Fax: 32-2274719

E-mail: normanmacmillan@hotmail.com 


\section{BIBLIOGRAFÍA}

1. Organización Mundial de la Salud. Estrategia Mundial sobre régimen alimentario, actividad física y salud. Ginebra. Suiza. 2004.

2. Vio F, Albala C. Epidemiología de la obesidad en Chile. Rev Chil Nutr 2000; 27: 97-112.

3. Muzzo S. Evolución de los problemas nutricionales en el mundo. El caso de Chile. Rev Chil Nutr 2002; 29 (2): 78-85.

4. Achten J. Optimizing fat oxidation through exercise and diet. Nutrition 2004; 20(7-8):716-27.

5. Horowitz J, Mora-Rodriguez R, Byerley L, Coyle E. Lipolytic suppression following carbohydrate ingestion limits fat oxidation during exercise. Am J Physiol 1997; 273 (Endocrinol Metab 36): E768-E755.

6. Bergman B, Brooks G. Respiratory gas-exchange ratios during graded exercise in fed and fasted trained and untrained men. J App Physiol 1999; 86: 479-487.

7. Achten J, Venables M, Jeukendrup A. Fat oxidation rates are higher during running compared with cycling over a wide range of intensities. Metabolism 2003; 52(6):747-752.

8. Coyle E, Jeukendrup A, Wagenmakers A, Saris W. Fatty acid oxidation is directly regulated by carbohydrate metabolism during exercise. Am J Physiol 1997; 273 (Endocrinol Metab 36): E268-E275.
9. Talanian J, Galloway S, Heigenhauser G. Two weeks of high-intensity aerobic interval training increases the capacity for fat oxidation during exercise in women. J Appl Physiol 2007; 102:14391447.

10. Al Mulla N, Simonsen L, Bulow J. Post exercise adipose tissue and skeletal muscle lipid metabolism in humans: the effects of exercise intensity. J Physiol 2000; 524(3):919-928.

11. Tremblay A, Simoneau J, Bouchard C. Impact of exercise intensity on body fatness and skeletal muscle metabolism. Metabolism 1994; 43 (7):814-818

12. Goto K, Ishii N, Yoshioka T, Takamasu K. Effects of resistance exercise on lipolysis during subsequent submaximal exercise. Med Sci Sports Exerc 2007; 39(2): 308-315.

13. Yoshioka M, Doucet E, St-Pierre S. Impact of high-intensity exercise on energy expenditure, lipid oxidation and body fatness. Internat J Obes 2001, 25:332-339.

14. Diaz E, Galgani J, Aguirre C. Glycaemic index effects on fuel partitioning in humans. Obesity Rev 2006. 7: 219-226.

15. Stevenson E, Williams C, Mash L. Influence of highcarbohydrate mixed meals with different glycemic indexes on substrate utilization during subsequent exercise in women. Am J Clin Nutr 2006; 84(2): 354-360. 